\title{
Nivel de competencia digital del alumnado de educación primaria en Galicia. La influencia sociofamiliar
}

\section{Digital competence of primary school students in Galicia. The socio- family influence}

\author{
Esther Martínez-Piñeiro \\ Universidade de Santiago de Compostela. Santiago de Compostela, España \\ esther.martínez@usc.es \\ Adriana Gewerc \\ Universidade de Santiago de Compostela. Santiago de Compostela, España \\ adriana.gewerc@usc.es \\ Ana Rodríguez-Groba \\ Universidade de Santiago de Compostela. Santiago de Compostela, España \\ ana.groba@usc.es
}

\begin{abstract}
Resumen
Se presenta resultados de la aplicación de una prueba de evaluación de la competencia digital en una muestra de alumnado de sexto de Educación primaria de la comunidad autónoma de Galicia en el curso 2018-19. La prueba se basó en el modelo DigComp adaptado a la edad de los sujetos. La muestra fue de tipo estratificada con afijación proporcional en función del número total de alumnos/as de $6^{\circ}$ de educación. Los datos fueron analizados con el programa SPSS, versión 22.0. Se llevaron a cabo análisis descriptivos univariables y pruebas de contraste de hipótesis. Los resultados permiten conocer el nivel del alumnado gallego en cada una de las cinco áreas de la competencia digital, tanto en conocimientos como en actitudes, así como las posibles diferencias entre ellos en función del hábitat, el centro y las características sociofamiliares. El nivel más bajo se obtiene en las dimensiones informacional y creación de contenidos. Mientras que la media más alta alcanzada es en el área de seguridad. El promedio global en el conjunto de la prueba es de 60 puntos (sobre 100).
\end{abstract}

Palabras clave: competencia digital, evaluación, educación primaria, DigComp

\begin{abstract}
Results of the digital competence's test is presented in a sample of sixth Primary Education students from the autonomous community of Galicia in the 2018-19 academic year. The test was based on the DigComp model adapted to the subjects' age. The sample was of stratified type with proportional affixation according to the total number of 6th grade students. The data were analyzed with the SPSS program, version 22.0. Univariate descriptive analyzes and hypothesis contrast tests were carried out. The results allow knowing the level of Galician students in each areas of digital competence, both in knowledge and attitudes, as well as the possible differences between them depending on the habitat, the school and the socio-family characteristics. The lowest level is obtained in the informational and content creation dimensions. The highest average reached is in the security area. The overall average of all dimensions is $60 / 100$.
\end{abstract}

Keywords: digital skills, primary school; DigComp 


\section{1- Introducción}

Las tecnologías digitales están consideradas, cada vez con mayor penetración, como un medio fundamental para superar las necesidades y fortalecer los derechos de los ciudadanos (tanto jóvenes como adultos), y ocupan un sitio privilegiado en la provisión de recursos, protección y participación social. Por lo tanto, las habilidades que se desarrollan para su uso creativo y pertinente son consideradas como la base de la fuente de empleo (OECD, 2014). Recientemente, este potencial ha sido vinculado a los 17 objetivos del Desarrollo Sostenible (ODS) que entró en vigencia en 2016. Muchas intervenciones relacionadas con la empleabilidad tienen como objetivo aumentar las competencias de alfabetización digital para acceder a servicios, beneficios, capacitación y prácticas laborales del siglo XXI (Lloyds Bank, 2016). Otras utilizan la alfabetización digital para abordar iniciativas de compromiso y bienestar social y como conducto para la participación en democracia o acceso a servicios públicos (Imison, Castle-Clarke, Watson \& Edwards (2016). Pero también han generado muchas preocupaciones por exacerbar las formas de exclusión, la amplificación de riesgos preexistentes, o la necesidad de frenar el estado avanzado de vigilancia y la explotación comercial a la que estamos sometidos. Lo cual hace más evidente el fundamental lugar de la educación para amplias capas de la población en el sostenimiento y profundización que ayude a las nuevas generaciones a saber enfrentarse a los nuevos problemas que estas tecnologías han traído consigo, sin por ello dar la espalda a todas sus potencialidades para el desarrollo humano.

En ese sentido, las políticas europeas e internacionales han empezado a incluir iniciativas educativas en torno a los nuevos medios y propuestas que regulan el uso de las tecnologías digitales en la educación. Tanto desde la OCDE, la UNESCO y la Unión Europea se han hecho eco de los cambios socio-tecnológicos que se han venido produciendo, y con ello, de la importancia de incorporar en la educación formal nuevos alfabetismos relacionados con los medios digitales. En el año 2006, en España se incluye por primera vez en el currículo de las enseñanzas obligatorias, ocho competencias básicas. Una de ellas es la de «Tratamiento de la información y competencia digital»(LOE, 2006), una competencia que se mantiene (aunque reformulada) en la modificación de esta ley en el año 2013 (competencia digital) (LOMCE, 2013). Además, en el año 2009, en colaboración con las comunidades autónomas, el gobierno lanza el proyecto Escuela 2.0 (Area \& Sanabria, 2014), que supone la introducción masiva de las tecnologías a las aulas de $5^{\circ}$ y $6^{\circ}$ de primaria y $1^{\circ}$ y $2^{\circ}$ de secundaria, a través del Modelo $1: 1$, un ordenador para cada niño/a. En Galicia el programa se denominó Abalar y luego se extendió a la integración del libro digital en un programa denominado Edixgal, que se define por establecer ambientes plenamente digitales para dar apoyo al proceso de enseñanza y aprendizaje relegando el papel del libro de texto tradicional, tal y como se ha entendido hasta ahora (Fraga-Varela y Alonso-Ferreiro, 2016).

La investigación ha dado cuenta en todos estos años que las estrategias y políticas desarrolladas hasta el momento fueron top-down, descontextualizadas $\mathrm{y}$ tecnológicamente deterministas (Gewerc y Montero, 2013; Sancho y Alonso-Cano, 2012) y con escaso alcance para la innovación de la enseñanza (Montero y Gewerc, 2013). También la investigación de los últimos años ya ha mostrado que niños, niñas y jóvenes no son "nativos digitales" (Livingston, 2017) ya que, a pesar que tienen contacto con las

Nivel de competencia digital del alumnado de educación primaria en Galicia. La influencia sociofamiliar. Esther Martínez, Adriana Gewerc y Ana Rodríguez.

Página 2 de 25 
tecnologías a edades cada vez más tempranas (UNICEF, 2017), necesitan de un amplio apoyo para desarrollar habilidades básicas, como la búsqueda de información especializada frente a la cantidad disponible, su análisis crítico decantando aquellas que son falsas o mal fundamentadas; la comunicación eficiente; o el necesario cuidado de los datos que se incorporan en la red conociendo su destino y propiedad y cómo prevenir situaciones de potencial peligro, dado que las tecnologías digitales están cada vez más en manos de grandes conglomerados y son una " caja negra", tratan los derechos, la privacidad y los intereses de sus usuarios, en función de sus intereses.

Son escasos los estudios que abordan los resultados en el aprendizaje de la competencia digital en la etapa de la educación primaria. Por lo tanto, lo que niñas, niños y jóvenes conocen del mundo digital, sigue siendo una gran incógnita y, generalmente, estos saberes no son reconocidos por el sistema educativo como fundamentales para la supervivencia $\mathrm{y}$, por lo tanto, poco valorados. De ahí que sea sumamente necesario desarrollar instrumentos que permitan conocer qué saben, cómo y dónde lo aprendieron para poder elaborar propuestas que partan de los conocimientos previos y de los intereses, necesidades y problemas que la juventud tiene en este momento histórico.

Para responder a esta necesidad, se desarrolló la investigación financiada por el Ministerio de Economía y Competitividad-Fondos FEDER (EDU2015-67975- C3-1- P), cuyos objetivos fueron identificar, analizar, evaluar y comprender la competencia digital (en adelante CD) de estudiantes de $6^{\circ}$ de Primaria (11-12 años); así como la influencia de este aprendizaje en los procesos de inclusión social en cuatro comunidades del territorio español (Galicia, Madrid, Castilla la Mancha y Castilla y León). En el estudio participó también la Universidad de Salamanca, como responsable de uno de los subproyectos de la investigación.

Para la consecución de esta meta se optó por un diseño mixto de tipo secuencial exploratorio organizado en dos grandes fases, la primera de ellas cualitativa, que se concretó en un estudio de casos múltiple, centrado en niños y niñas de sexto curso de educación primaria, y una segunda parte de tipo cuantitativo que se concretó en el diseño y aplicación de una prueba de evaluación de la competencia digital en una muestra de alumnado de sexto de educación primaria de la comunidad autónoma de Galicia. Además, se recogió información de tipo sociodemográfico y de usos de las tecnologías en los hogares de los sujetos.

En este artículo se presentan parte de los resultados de la segunda fase del estudio, centrándonos en los datos generales obtenidos en la prueba de competencia digital, su relación con algunas variables sociodemográficas y con el uso de las tecnologías en el hogar.

\section{Competencia digital en contexto}

Los modelos de alfabetización digital que se han desarrollado en los últimos veinte años incluyen conocimientos, habilidades y competencias. El conocimiento como la información consciente y la comprensión que los usuarios tienen de la existencia y el uso de diferentes herramientas digitales. Las habilidades digitales, podrían tener una vertiente más práctica, medible en la aplicación de ciertos conocimientos y aptitudes en este uso. Y, por último, la competencia se presenta como la habilidad para aplicar tanto el

Nivel de competencia digital del alumnado de educación primaria en Galicia. La influencia sociofamiliar. Esther Martínez, Adriana Gewerc y Ana Rodríguez.

Página 3 de 25 
conocimiento como las habilidades en diferentes contextos (en lo profesional y en la vida personal). La alfabetización digital compila tanto la conciencia, como las habilidades prácticas y las competencias necesarias para que los usuarios accedan, comprendan, evalúen, se comuniquen con otros y creen contenido digital de manera estratégica hacia el logro de sus objetivos profesionales y personales (Lordache, Mariën y Baelden, 2017).

La investigación que aquí se presenta utiliza como base el modelo DigComp de la Unión Europea que, desde su primera publicación, en 2013, se ha convertido en referencia para la planificación de estrategias de desarrollo de la competencia digital de todos los estados miembros. En su modelo conceptual la define como:

Un conjunto de conocimientos, habilidades, actitudes, estrategias y valores que son puestos en acción cuando usamos las tecnologías y los medios digitales para realizar tareas, resolver problemas, comunicarse, tratar información, colaborar, crear y compartir contenidos y crear conocimiento, de forma efectiva, eficiente, apropiada, crítica, creativa, autónoma, flexible, ética y reflexiva para el trabajo, el ocio, la participación, el aprendizaje, la socialización, el consumo y el empoderamiento. (Ferrari, 2012, p.30)

Comprende cinco áreas (información, comunicación, resolución de problemas, creación de contenidos y seguridad) y 21 subdimensiones traducidas en tres niveles de logro (básico, intermedio y avanzado). En 2016 JRC publica el DigComp 2.0, con actualización de terminología y del modelo conceptual, y con ejemplos de implementación del modelo en los diferentes estados de la región. La versión más actual es el DigComp 2.1, centrado en la expansión de los iniciales tres niveles de logro hacía ocho (Vuorikari, Punie, Carretero, y Van den Brande, 2016; 2017). Está dirigida a la ciudadanía y por tanto es necesario realizar una adaptación a las características etarias y contextuales de los sujetos que fueron objetos de investigación.

Es importante destacar que, a pesar de que el acceso se ha generalizado con diferencias entre regiones pobres y ricas (World Bank, 2016), la investigación da cuenta de que aún existen otras lagunas en relación a la alfabetización digital y las habilidades o la capacidad de obtener capacitación o experiencia con las tecnologías, por lo tanto, incluso cuando existen oportunidades, no todos tienen las mismas posibilidades de aprovechar las potencialidades ni de estar prevenidos en relación a los riesgos (Livingstone, Haddon y Görzig, 2012; Livingston, Nandi, Banaji y Stoilova, 2017). Las disparidades en relación a los usos de los medios digitales ocurren en paralelo e incluso a veces profundizan otras desigualdades preexistentes, creando así múltiples desventajas entre y dentro de los países, regiones y áreas (UNICEF, 2013). Maximizar los potenciales positivos de las tecnologías requiere una buena comprensión de cómo interactúa con factores sociales, políticos y económicos importantes para el desarrollo (Banaji, 2017; World Bank, 2016). Por lo tanto, a la hora de analizar el desarrollo de la competencia digital, se hace necesario evitar las homogeneizaciones que eliminan las diferencias sociales, culturales y económicas y visualizarla en el contexto de niños y adolescentes estudiados. Solo algunos estudios prestan atención a la importancia de lo social, la clase y el género como ejes principales de la desigualdad y, sin analizarla, no es posible pensar en propuestas de inclusión. De allí que haya sido necesario incluir, en los instrumentos utilizados para la investigación, dimensiones socioculturales que permitan identificar cuáles son las

Nivel de competencia digital del alumnado de educación primaria en Galicia. La influencia sociofamiliar. Esther Martínez, Adriana Gewerc y Ana Rodríguez. 
prácticas con tecnología que los sujetos desarrollan. Esto posibilitó profundizar en las diferencias para obtener un conocimiento más profundo de la realidad estudiada.

Así como la transición a la cultura popular masiva trajo beneficios y amenazas a las personas, las tecnologías digitales y las nuevas alfabetizaciones podrían generar aportaciones importantes a las comunidades "desfavorecidas", aunque también retos y riesgos que hay que analizar.

\section{Evaluación de la competencia digital}

Los estudios previos que se centraron en la evaluación de la competencia digital se diferencian porque ponen el foco en un aspecto o dimensión o por el tipo de herramientas que utilizan: autopercepción del sujeto o evaluación. En el primer caso, se tiene en cuenta la opinión del sujeto sobre sus propias habilidades relacionadas con la competencia digital. En el caso de las que evalúan, se busca medir el nivel de desarrollo de la competencia abordada.

Son numerosos los estudios de autopercepción que trabajan la dimensión informacional de la CD. Por ejemplo, el trabajo de Rodríguez, Olmos y Martínez (2012) analiza la autopercepción a través de una escala de evaluación denominada IL-HUMASS que se aplica en el ámbito del alumnado universitario a partir de una adaptación del trabajo de Pinto (2009) con el objetivo de comprobar las evidencias de validez y fiabilidad. Así mismo, algunos trabajos abordan la evaluación de la competencia mediática en la Educación Infantil mediante un cuestionario que permite establecer tres niveles de competencia: excelente, aceptable y mínimo (García, Duarte y Guerra, 2014). También se incluye en esta línea el estudio desarrollado por Ferrés, et. Al. (2011) que aborda el análisis de la competencia mediática en el conjunto de la ciudadanía a través de una investigación a nivel nacional mediante la aplicación de cuestionarios evaluadores, entrevistas y grupos de discusión. Algunas de las conclusiones de este trabajo son, por ejemplo, que la utilización de nuevas tecnologías se encuentra condicionada, más que por la edad o los conocimientos previos, por la existencia de una motivación poderosa que lleve a interesarse por ellas, cuestión en la que también insisten Van Deursen y Van Dijk (2011). El test ADO (Alfabetización digital on-line) analiza la competencia mediática, y como señalan algunas investigaciones, ha permitido mostrar que el nivel de la ciudadanía no es el esperado (Ferrés et al., 2011), además de evidenciar una brecha digital entre generaciones y de género. Según este estudio el perfil medio de usuario de internet está más involucrado hacia el ocio y a consumir contenidos que a producir y crear.

González, Espuny, de Cid y Gisbert (2012) diseñaron un instrumento para la educación secundaria y con esta base lo ampliaron a los estudios de grado Gisbert, Espuny y González (2011). Buscan poder "incidir directamente en aquellos ámbitos en los que los grupos de estudiantes presenten un menor nivel de competencia" (González, Espuny, de Cid y Gisbert, 2012, p.301). Dentro de esta línea de investigación, se crea un cuestionario orientado a jóvenes en riesgos de exclusión social (Carrera, Vaquero y Baslells (2011). En otra comunidad autónoma Castilla-León, se crea un instrumento de escala tipo Likert que busca abordar las áreas competenciales del DigComp: información, comunicación, creación de contenidos, seguridad y resolución de problemas, midiendo la competencia

Nivel de competencia digital del alumnado de educación primaria en Galicia. La influencia sociofamiliar. Esther Martínez, Adriana Gewerc y Ana Rodríguez.

Página 5 de 25 
digital del alumnado en la etapa de Educación Primaria (Pérez-Escoda, CastroZubizarreta y Fandos-Igado, 2016)

Entre los trabajos que se centran en la evaluación de la Competencia Digital, es útil la clasificación que ofrece Oakleaf (2008) en base a tres principales tendencias: test de respuesta única, pruebas de rendimiento y rúbricas. Van Deursen y Van Dijk (2011) ponen de manifiesto en su estudio de la población holandesa las estrechas relaciones entre bajo nivel de competencia digital e indicadores de exclusión social, lo que evidencia el valor de los procesos educativo en el entorno cultural de los jóvenes (Van Deursen y Van Diepen, 2013). Se pone de relieve que los niños/as de Educación Primaria utilizan estrategias inadecuadas cuando buscan información y no combinan la información para tomar decisiones beneficiosas (Van Deursen, Görzig, Van Delzen, Perik y Stegeman, 2014). Destaca, también, la herramienta de evaluación iSkills desarrollada por el Educational Testing Service (ETS), cuyo objetivo estuvo centrado en las habilidades de los estudiantes universitarios a la hora de investigar, organizar y comunicar la información con tecnología (Katz, 2007). Destacan, en la misma línea, el Information Literacy Test (ILT) (Cameron, Wise y Lottridge, 2007), los cuestionarios de James Madison University's Information Literacy Test (Fain, 2011) y el Instant DCA (Calvani, Fini, Ranieri y Picci, 2012).

Estaríamos entonces ante factores a los que, por tanto, es preciso atender y analizar de un modo integral a la hora de aproximarnos con rigor al análisis de la competencia digital de los estudiantes. Es de resaltar que los trabajos que analizan y evalúan la $\mathrm{CD}$, en general lo hacen centrándose sólo en alguna de sus dimensiones, por lo que no se percibe una visión completa de esta competencia que es sumamente compleja. Aunque se constata el valor de analizarla en el nicho cultural en donde se desarrolla, y el peso e influencia del entorno sociofamiliar, son escasos los trabajos que estudian esta competencia en la educación obligatoria y, en ese contexto, los trabajos que abordan la problemática en el nivel de educación primaria. Se visualiza entonces la necesidad de conocer en profundidad qué habilidades desarrollan estos niños/as, cómo y cuándo lo hacen, quienes están influyendo en ese desarrollo, contrastarlo con las perspectivas teóricas que proponen los expertos en esta temática y con ello construir una prueba que ayude a realizar diagnósticos más atinados con la realidad social en donde los sujetos viven y se desarrollan. Cuestión que ha sido objeto de la investigación que tiene como base el presente artículo.

\section{Objetivos y metodología}

La investigación ha tenido como objetivo -tal y como se ha mencionado- identificar, analizar, comprender y evaluar la competencia digital que poseen y utilizan en su vida cotidiana los estudiantes de enseñanza obligatoria y la posible influencia de esta competencia en los procesos de inclusión social. El trabajo se desarrolló en una primera fase, cualitativa, en la que se ha indagado, a través de un estudio de casos múltiple, en los procesos de apropiación de las tecnologías por los preadolescentes y en el papel de la familia y los amigos. Esta etapa permitió generar indicadores adaptando la propuesta de DigComp a la población objeto de estudio. La segunda parte, que se desarrolla en este trabajo, ha tenido como objeto la evaluación de la competencia digital.

Nivel de competencia digital del alumnado de educación primaria en Galicia. La influencia sociofamiliar. Esther Martínez, Adriana Gewerc y Ana Rodríguez. 
Se ha empleado, por lo tanto, un diseño mixto secuencial exploratorio (Creswell y Plano Clark, 2007; NIH Office of Behavioral and Social Sciences, 2018). En este tipo de diseños los hallazgos de la fase cualitativa son necesarios para llevar a cabo el estudio cuantitativo, y los resultados obtenidos en esta fase permiten confirmar o generalizar hallazgos cualitativos (Pluye y Hong, 2014).

\section{Población y muestra}

La población de estudio está configurada por el alumnado de $6^{\circ}$ curso de educación primaria de centros públicos de la comunidad autónoma gallega en el curso 2018-19, pero dado que en el momento de realizar el trabajo de campo no se conocía este dato con exactitud se tomó como referencia el dato provisional (Xunta de Galicia, 2017) del alumnado de $5^{\circ}$ curso de educación primaria en este tipo de centros en el curso 2017-18, en concreto:

\section{Tabla 1}

Datos alumnado en $5^{\circ}$ de Educación Primaria en el curso 2017-2018

\begin{tabular}{ccccc}
\hline A Coruña & Lugo & Ourense & Pontevedra & total \\
\hline $\mathbf{6 . 7 6 6}$ & 1.666 & 1.428 & 6.025 & $\mathbf{1 5 . 8 8 5}$ \\
\hline
\end{tabular}

Fuente: Datos y cifras de la enseñanza no universitario. Xunta de Galicia curso 2107-18

Para el cálculo inicial de la composición de la muestra se empleó un muestreo de tipo estratificado con afijación proporcional en función del número de alumnos/as de $6^{\circ}$ de Educación Primaria en cada uno de los estratos. Se emplearon como niveles del muestreo el tipo de provincia donde está el centro al que pertenece el sujeto (clasificándolas según su ubicación geográfica en atlántica o no atlántica); el tipo de ayuntamiento en el que está ubicado el centro en función de su densidad poblacional (densamente poblado, medianamente poblado y poco poblado, atendiendo a la clasificación empleada por el Instituto Galego de Estatística), y la inclusión o no del centro en el programa de inmersión tecnológica existente en Galicia en este curso denominado Edixgal (Educación Digital en Galicia).

Para la selección de los centros participantes se optó por utilizar una estrategia intencional de cara a garantizar la fiabilidad de la muestra, también para mantener un conjunto de criterios de eficiencia en la realización del trabajo de campo. En concreto:

- Cierta proximidad del centro

- Expectativas de que participen en el proceso.

La muestra esperada quedó inicialmente compuesta por 764 escolares, si bien por diversas circunstancias (falta de tiempo en el centro escolar, fallos de la aplicación, ausencia del alumno/a, etc.), no todos ellos realizaron la prueba de forma completa, por lo que -como se observará- el tamaño muestral ofrecido en los resultados de la prueba es inferior.

Nivel de competencia digital del alumnado de educación primaria en Galicia. La influencia sociofamiliar. Esther Martínez, Adriana Gewerc y Ana Rodríguez. 
La distribución geográfica contemplada en el diseño de la muestra, indica que el $70 \%$ de los sujetos residen en provincias atlánticas (Pontevedra o Coruña) y el $30 \%$ en no atlánticas (Lugo e Ourense), mucho menos pobladas.

Atendiendo a la densidad poblacional del ayuntamiento en el que se sitúa el centro escolar, el 42,5\% del alumnado estudia en centros de las grandes ciudades, el $37 \%$ de villas y el resto en pequeñas poblaciones.

Un $37,4 \%$ de los estudiantes lo hacen en centros que participan en el programa autonómico de inmersión tecnológica Edixgal y un 62,6\% en aquellos que no lo hacen, manteniendo una proporción similar a la aplicación de este programa en la población de referencia.

La distribución en cuanto al género es bastante homogénea, el 47,4\% son niños y el 52,6\% son niñas, mayoritariamente, como cabe esperar por el curso en el que están, de 12 años $(84 \%)$

\section{Tabla 2}

Nivel de estudios de los padres/madres

\begin{tabular}{lcc}
\hline & Madre & Padre \\
\hline No estudió & $0,6 \%$ & $1 \%$ \\
Educación G Básica o EP & $13,2 \%$ & $22 \%$ \\
ESO & $8,5 \%$ & $11 \%$ \\
FP & $21,4 \%$ & $25,4 \%$ \\
Bachillerato & $17,1 \%$ & $14,3 \%$ \\
Universidad & $39,3 \%$ & $26,4 \%$ \\
\hline
\end{tabular}

Fuente: Elaboración propia

Como puede verse en la tabla, el 39,3\% de las madres de la muestra tiene estudios universitarios y el $21,4 \%$ de formación profesional. En su encuesta estructural a hogares de 2017, el Instituto Galego de Estadística expone un porcentaje un poco menor $(24,70$ $\%$ de los hombres y el $26,86 \%$ de las mujeres) aunque se mantienen las diferencias. La distribución geográfica de las familias teniendo en cuenta su nivel académico -como cabía esperar- no es homogénea; en las zonas urbanas hay más hijos de madres y padres con estudios universitarios y menos sin estudios o con estudios primarios, mientras que en las zonas rurales ocurre lo contrario.

\section{Instrumentos}

La prueba de evaluación de la CD fue diseñada por el grupo de investigación GITE de la Universidad de Salamanca -responsable de uno de los proyectos coordinados-, a partir del modelo de Competencia propuesto por DIGCOMP. Se llevó a cabo una primera adaptación del mismo teniendo en cuenta la edad y las características de los destinatarios. De este modo se definió un modelo de indicadores previos que incluía las 5 áreas del modelo DIGCOM e ítems referidos a conocimientos, capacidades y actitudes.

Nivel de competencia digital del alumnado de educación primaria en Galicia. La influencia sociofamiliar. Esther Martínez, Adriana Gewerc y Ana Rodríguez. 
Posteriormente se procedió a la validación externa de este modelo de indicadores por parte expertos de diferentes niveles educativos. El nivel de acuerdo entre jueces se llevó a cabo siguiendo el modelo de Lawshe (1975) y la revisión de Tristán-López (2008). El número de jueces que valoró cada área superó el número mínimo indicado en el modelo, contando con más de 10 jueces en todos los casos.

En base a la información obtenida, tanto de carácter cuantitativo como cualitativo y tras esta revisión, se llevó a cabo una depuración del modelo considerando las apreciaciones realizadas polo conjunto de expertos, de modo que se contase con un modelo definitivo, a partir del que diseñar los ítems que conforman la prueba. En este modelo se incorporaron además, indicadores extraídos de los estudios de caso realizados en la primera fase de la investigación.

La versión inicial de la prueba fue mejorada a partir del análisis de los resultados de la aplicación piloto llevada a cabo en el segundo semestre del curso 17-18.

\section{Tabla 3}

Número de ítems que conforman la prueba según conocimientos y capacidades, y actitudes, atendiendo a las subdimensiones

\begin{tabular}{cccc}
\hline & $\begin{array}{c}\text { Conocimientos } \\
\text { Y capacidades }\end{array}$ & Actitudes & Total \\
\hline Información & 12 & 6 & 18 \\
\hline Comunicación & 18 & 6 & 24 \\
\hline Creación de contenidos & 16 & 6 & 22 \\
\hline Seguridad & 16 & 6 & 22 \\
\hline Resolución de problemas & 16 & 6 & 22 \\
\hline & 32 & 30 & 108 \\
\hline
\end{tabular}

Fuente: Elaboración propia

Las puntuaciones en la prueba para cada sujeto en cada una de las áreas son el resultado de sumar el número de respuestas correctas en los ítems de conocimientos y capacidades y en las preguntas actitudinales, tras llevarse a cabo la dicotomización de estas últimas. Se ha obtenido, por lo tanto, para cada uno de ellos una puntuación directa en conocimientos y capacidades, una puntuación directa en actitudes en cada área, y una puntuación directa final por área resultado de la suma de ambas puntuaciones. Dado que el número de ítems no es el mismo en todas las áreas, estas puntuaciones fueron normalizadas en base 20 para poder establecer comparaciones entre áreas. Además, se calculó una puntuación final en la prueba resultado de la suma de las puntuaciones en cada una de las área, tanto directa (máximo 108 puntos) como a partir das puntuaciones normalizadas, con 100 como puntuación máxima.

La fiabilidad de la prueba en términos de consistencia interna Alfa de Cronbach muestra valores moderadamente adecuados en cada una de las áreas y ligeramente superior para el conjunto del instrumento, que obtiene una fiabilidad de 0,89 , considerada alta atendiendo a los criterios habituales (Corral, 2009; George y Mallery, 2006). Además de esta prueba, se diseñó un cuestionario ad hoc para las familias de los niños y niñas, centrado en cuestiones de tipo sociodemográfico y posesión y uso de dispositivos

Nivel de competencia digital del alumnado de educación primaria en Galicia. La influencia sociofamiliar. Esther Martínez, Adriana Gewerc y Ana Rodríguez. 
digitales que nos permite poder establecer posibles relaciones entre características sociofamiliares (culturales, sociales y económicas), tipo de dispositivos, tiempo de usos y competencia digital.

\section{Análisis de datos}

Los datos fueron analizados con el programa SPSS, versión 22.0. En una primera etapa se llevaron a cabo análisis descriptivos univariables, centrados en conocer la distribución de las principales variables de estudio, (porcentajes, medias, desviación típica), así como análisis correlacional bivariado( Phi, Tau de Kendall), que nos permitieran conocer la posible asociación entre ellas Se realizaron también pruebas de contraste de hipótesis para analizar la posible diferencia entre grupos de alumnos, en concreto la prueba paramétrica t de Student, cuando se trataba de dos grupos y Anova de un factor cuando eran más; el nivel de significación utilizado es de 0,05.

\section{Resultados y Discusión}

Los datos recogidos han permitido acercarse a la realidad de los preadolescentes gallegos y al uso de las tecnologías en su hogar, al mismo tiempo que disponer de una medición de su competencia digital en conocimientos y capacidades, y actitudes, en cada una de las áreas, y de forma global, a través de la suma de la calificación en las cinco áreas.

\section{Dispositivos y su uso}

Existe una alta disposición de ordenador (92,3\%), teléfono móvil (89\%) y acceso a internet en los hogares (93\%), por lo que se desprende que la dificultad de acceso a los medios es reducida, en consonancia con los resultados ofrecidos por el INE (2018) (el 92,4\% de niños de 12 años españoles tienen acceso a Internet desde el hogar y el 75,1\% tiene teléfono móvil). A nivel internacional se observa que el creciente número de medios digitales disponibles es cada vez más accesible, asequible y personalizado, aunque persisten considerables desigualdades inter e intra regionales (Kleine et al.,2014; UNECOSOC, 2015; WEF, 2015;). Estas desigualdades están expresadas en el tipo de dispositivo, la calidad de la velocidad en el acceso a internet y en los modos de apropiación de los dispositivos.

La videoconsola también tiene una presencia destacada, pues el $75 \%$ de las familias tienen alguna, aunque observamos diferencias de género, ya que este porcentaje asciende a más del $86 \%$ de los hogares de los varones, mientras que en el de las mujeres es del $65 \%$.

Otros dispositivos tecnológicos no son tan usuales, como se recoge en la tabla 1. De todos ellos es el lector de libros digitales el que está presente en menos hogares, en un $26 \%$.

\section{Tabla 4}

Dispositivos tecnológicos en los hogares

\begin{tabular}{lc}
\hline Dispositivo & \% de sujetos que dispone de él en casa \\
\hline Televisión & $99,2 \%$ \\
Internet & $93 \%$ \\
Ordenador & $92,3 \%$ \\
Teléfono con conexión a internet & $89,2 \%$ \\
\hline
\end{tabular}

Nivel de competencia digital del alumnado de educación primaria en Galicia. La influencia sociofamiliar. Esther Martínez, Adriana Gewerc y Ana Rodríguez. 


\begin{tabular}{lc}
\hline Tableta & $82 \%$ \\
Videoconsola & $74,9 \%$ \\
Reproductor de música portátil & $47,1 \%$ \\
Canales de Tv de pago & $41 \%$ \\
Lector de libros digitales & $25,9 \%$ \\
\hline
\end{tabular}

Fuente: Elaboración propia

Los datos evidencian diferencias en los días en que se usan los dispositivos (los fines de semana frente a los días de escuela). Así, y según la información que nos proporciona el padre y la madre, de lunes a viernes un $60 \%$ no emplean el móvil y en un porcentaje cercano se señala que no juegan con la videoconsola. La televisión continúa siendo un dispositivo cotidiano, más del $70 \%$ la ve cuatro o cinco días y sólo un $4,2 \%$ no la ve nunca de lunes a viernes, es decir que entre los preadolescentes hay un consumo habitual de televisión también en los días de escuela. El televisor tiene presencia en el 99,1 \% según el INE (2018). Investigaciones previas (Del Moral y Villalustre, 2010) ponen de manifiesto que la dedicación de los escolares a la televisión y videojuegos ocupa más del $38 \%$ de su tiempo libre. Pero esto no es paralelo al conocimiento de los códigos del lenguaje audiovisual ya que niños y niñas son consumidores mayoritariamente pasivos, lo que les sitúa en un escenario de total desamparo ante los continuos mensajes que de ellos van recibiendo (López-Mondéjar, 2018).

El ordenador también tiene un alto porcentaje de uso, ya que cerca del $75 \%$ de los preadolescentes lo emplea de lunes a viernes, por ser un dispositivo cada vez más habitual para la realización de tareas escolares en este nivel de la enseñanza primaria.

\section{Tabla 5}

Frecuencia con la que utilizan determinados dispositivos entre semana (de lunes a viernes)

\begin{tabular}{lcccc}
\hline & Nunca & $\begin{array}{c}\text { 1 día por } \\
\text { semana }\end{array}$ & $\begin{array}{c}\text { Entre dos y tres } \\
\text { días }\end{array}$ & $\begin{array}{c}\text { Entre cuatro y cinco } \\
\text { días }\end{array}$ \\
\hline Tel. móbil & $60 \%$ & $7,5 \%$ & $14,4 \%$ & $18 \%$ \\
Tableta & $47,9 \%$ & 16,8 & $17,1 \%$ & $18,2 \%$ \\
Ordenador & $25,8 \%$ & $22,8 \%$ & $20,3 \%$ & $31 \%$ \\
Videoconsola & $57,8 \%$ & $22,9 \%$ & $12,7 \%$ & $6,5 \%$ \\
Televisión & $4,2 \%$ & $6,6 \%$ & $17,9 \%$ & $71,3 \%$ \\
\hline
\end{tabular}

Fuente: Elaboración propia

Las pautas de uso parecen diferir entre niños y niñas, especialmente respecto a la consola, el móvil y el ordenador, donde se han encontrado diferencias significativas. Claramente ellos le dedican mucho más tiempo que ellas a la videoconsola; esta resulta ser una actividad mucho más cotidiana para los varones. Frente a un $44 \%$ que dicen no usarla de lunes a viernes, hay un $70 \%$ de niñas que tampoco lo hacen. Estas en cambio hacen un mayor uso del teléfono móvil, ya que lo emplean como mínimo un día a la semana el $46 \%$ de las niñas, frente al 34\% de los niños. También hay un mayor porcentaje de niñas que

Nivel de competencia digital del alumnado de educación primaria en Galicia. La influencia sociofamiliar. Esther Martínez, Adriana Gewerc y Ana Rodríguez. 
de niños que usan cuando menos uno de los días el ordenador, en concreto un $78 \%$ frente a un $69 \%$.

Estos datos podrían ayudarnos a pensar en la influencia que tienen determinados dispositivos en el desarrollo de la competencia digital de los sujetos. Los videojuegos proveen a los jóvenes de recursos simbólicos para construir o expresar su propia identidad (Buckingham, 2008), al mismo tiempo que ofrecen la posibilidad de acceder a nuevas formas de lectura y escritura (Jones y Hafner, 2012). Más allá de una influencia corrupta o una pérdida de tiempo (Carbonell, 2014; Marco y Chóliz, 2014), se evidencia como una oportunidad para el crecimiento cognitivo y la práctica de la resolución de problemas (Lacasa, 2011; Sedeño, 2010). Que los varones jueguen más con este dispositivo, nos podría dar pistas de las habilidades que potencialmente desarrollan con esta práctica.

Se observa también que los hábitos de uso del ordenador en días escolares son diferentes en función del tipo de centro al que se asiste, siendo mucho más alto en aquellos que participan en el programa de inmersión tecnológica.

\section{Competencia digital}

Los resultados de la prueba permiten conocer el nivel del alumnado gallego en cada una de las áreas de la competencia digital, tanto en conocimientos como en actitudes, así como las posibles diferencias entre ellos en función del hábitat, el centro y las características sociofamiliares.

Como puede observarse, atendiendo a las puntuaciones normalizadas a 20, la puntuación media más alta alcanzada es en el área de seguridad $(13,09)$, frente al área de creación de contenidos, que apenas sobrepasa los 11 puntos. Puntuaciones medias similares se alcanzan en el área de resolución de problemas e informacional, 11,5 y 11,53 respectivamente.

\section{Tabla 6}

Muestra, puntuaciones medias alcanzadas y desviaciones típicas en las distintas áreas

\begin{tabular}{lcrr}
\hline & N & Media & D.T \\
\hline Área informacional & 618 & 11,54 & 3,20 \\
Área comunicacional & 602 & 12,68 & 3,30 \\
Area creación de contenidos & 585 & 11,13 & 2,96 \\
Área de seguridad & 563 & 13,09 & 3,54 \\
Resolución de problemas & 537 & 11,50 & 3,07 \\
Total (base 100) & 521 & 60,08 & 12,94 \\
\hline
\end{tabular}

Fuente: Elaboración propia.

Al "descomponer" esta puntuación atendiendo a los dos tipos de ítems (conocimientos y actitudes) el orden entre ellas se mantiene, pero solo en dos se superan los siete puntos de

Nivel de competencia digital del alumnado de educación primaria en Galicia. La influencia sociofamiliar. Esther Martínez, Adriana Gewerc y Ana Rodríguez. 
los catorce posibles en conocimientos, es decir el alumnado sólo aprueba en conocimientos de las áreas comunicacional y seguridad. Es el aspecto actitudinal el que "eleva" el nivel del alumnado. Es llamativo que precisamente en conocimientos del área informacional la puntuación media no alcance el aprobado cuando es habitualmente la más trabajada en el contexto escolar (Alonso-Ferreiro, 2016), por ser una de las competencias básicas de la enseñanza primaria, en contraposición al área de seguridad, que tiene escasa presencia en el curriculum escolar. Esto podría estar indicando que la mayor parte de estas habilidades se adquieren fuera de la escuela, en el llamado "tercer espacio" (McDougall, Readman y Wilkinson, 2018)

\section{Tabla 7}

Media y desviación típica en cada área, en conocimientos y en actitudes

\begin{tabular}{lrrrrr}
\hline & \multicolumn{3}{c}{ Conocimientos } & \multicolumn{2}{c}{ Actitudes } \\
\hline & $\mathrm{N}$ & Media & DT & Media & DT \\
\hline Área informacional & 630 & 6,76 & 2,61 & 4,72 & 1,29 \\
Área comunicacional & 615 & 7,59 & 2,64 & 5,05 & 1,29 \\
Área creación de contenidos & 603 & 6,45 & 2,37 & 4,60 & 1,36 \\
Área de seguridad & 571 & 7,95 & 2,85 & 5,09 & 1,28 \\
Resolución de problemas & 550 & 6,77 & 2,52 & 4,66 & 1,48 \\
\hline
\end{tabular}

Fuente: Elaboración propia

Si tenemos en cuenta el nivel alcanzado por el alumnado (muy bajo, bajo, medio y alto), atendiendo a la puntuación obtenida, se observan importantes variaciones en función del área competencial; un $20 \%$ presenta un nivel bajo o muy bajo en seguridad, porcentaje que llega al $34,4 \%$ en creación de contenidos y al $29 \%$ en competencia informacional. Estos bajos resultados representan un buen punto de partida para hacer un análisis y proyectar soluciones a la problemática.

Es importante visualizar los datos teniendo en cuenta aspectos contextuales que podrían estar influyendo en su concreción. El nivel competencial parece mediado por diversas variables tanto personales como del entorno de los jóvenes; como veremos a continuación, el género, el nivel académico de la familia y algunos hábitos de los adolescentes tienen relación con los resultados alcanzados en la prueba, así también el tipo de centro y su ubicación. Sin embargo, están evidenciando la necesidad de un trabajo en profundidad sobre aspectos que las instituciones educativas necesitan analizar.

Nivel de competencia digital del alumnado de educación primaria en Galicia. La influencia sociofamiliar. Esther Martínez, Adriana Gewerc y Ana Rodríguez. 


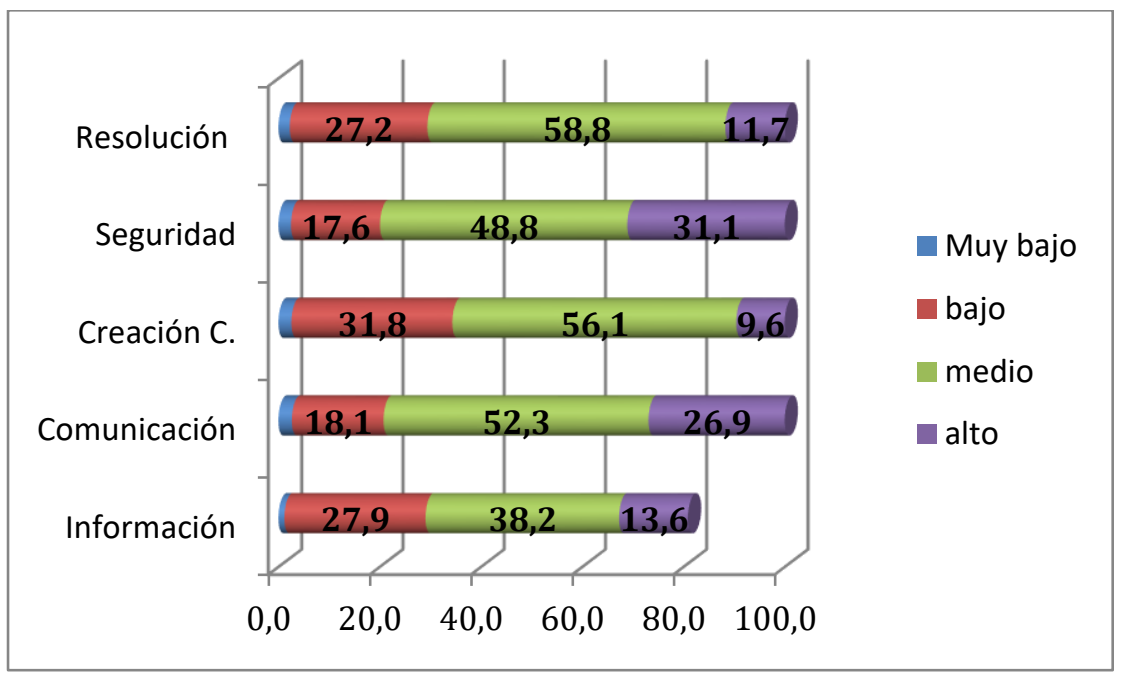

Figura 1.

Porcentaje de alumnos/as en cada nivel competencial por área

Fuente: Elaboración propia

\section{Variables personales y familiares}

Las diferencias entre niños y niñas no sólo están presentes en los hábitos de uso, sino también en los resultados de la prueba, en la que, en promedio las chicas obtienen puntuaciones más altas en todas las áreas siendo significativas en comunicación, creación de contenidos y seguridad (significación bilateral de 0,001;0,008;0,001 respectivamente), y también en el conjunto de la prueba con una puntuación media de 66,4 (sobre 100) frente a 62,6. Resultados que coinciden con los encontrados en otros estudios del contexto español (Pérez-Escoda, Castro-Zubizarreta y Fandos-Igados, 2016)

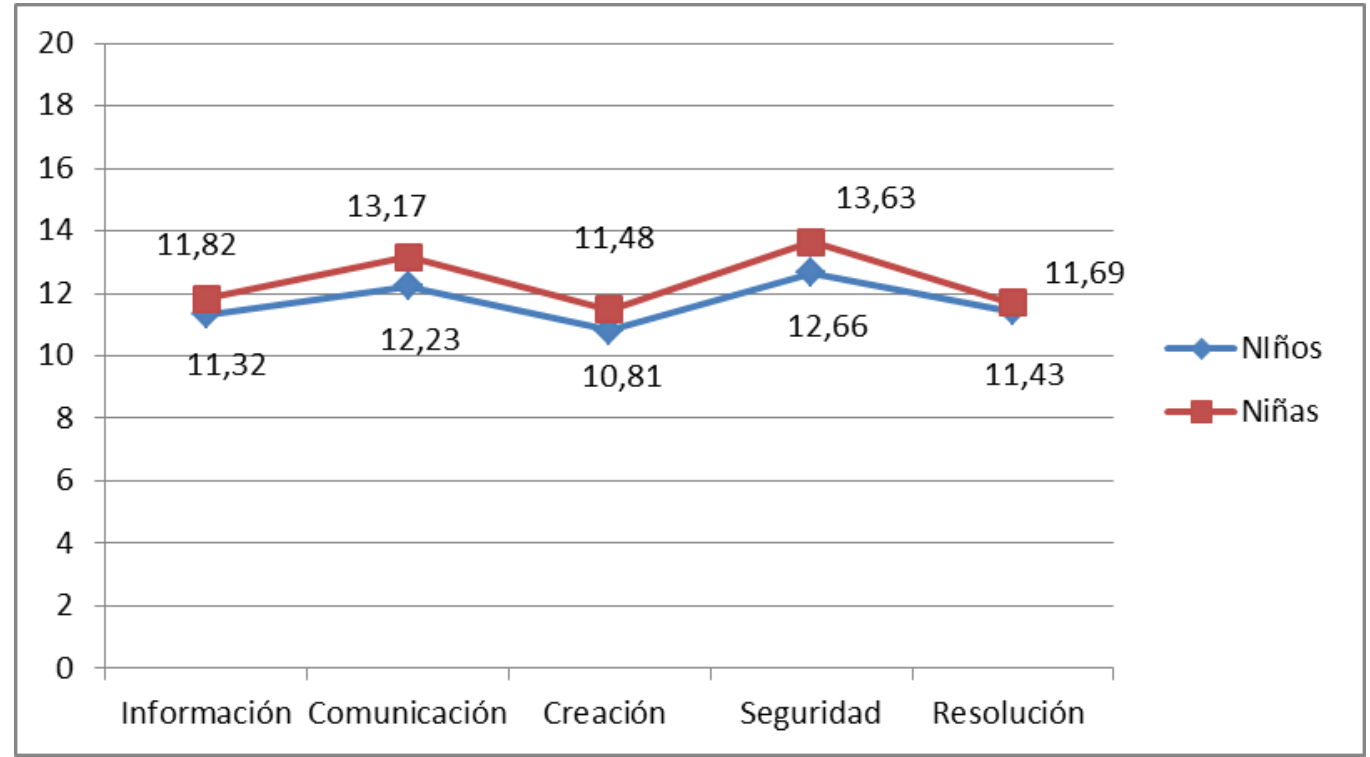

Figura 2:

Nivel de competencia digital del alumnado de educación primaria en Galicia. La influencia sociofamiliar. Esther Martínez, Adriana Gewerc y Ana Rodríguez. 
Media de los niños y niñas en las distintas áreas de la competencia

Fuente: Elaboración propia

Estas diferencias sin embargo disminuyen si tenemos en cuenta el hábitat del centro, ya que en zonas rurales las diferencias entre ellos y ellas dejan de ser significativas y en las villas sólo se mantiene en el aspecto informacional. En las zonas urbanas son las niñas las que superan significativamente a sus compañeros. Esta situación puede estar dada porque la mayor parte de los centros que participan de los programas de inmersión tecnológica están situados en las grandes ciudades. Tal como muestra el estudio de FragaVarela y Alonso-Ferreiro (2016) las políticas y las fases de implementación del proyecto Edixgal estuvieron polarizadas claramente hacia los grandes núcleos de población de la Comunidad Autónoma. De hecho, las diferencias entre niñas y niños en el total de la prueba dejan de ser significativas en los centros inmersos en este programa, pero no así en aquellos que no lo están (significación 0,001).

No se observaron diferencias significativas en función de los estudios del padre, pero sí en lo relativo a los de la madre. Así es en las dimensiones informacional y comunicacional, donde los hijos/as de universitarias presentan cualificaciones significativamente más altas que en los casos en que no tiene estudios o son primarios y las de estudios medios. Un indicador del capital cultural de las familias (Bourdieu, 1986)

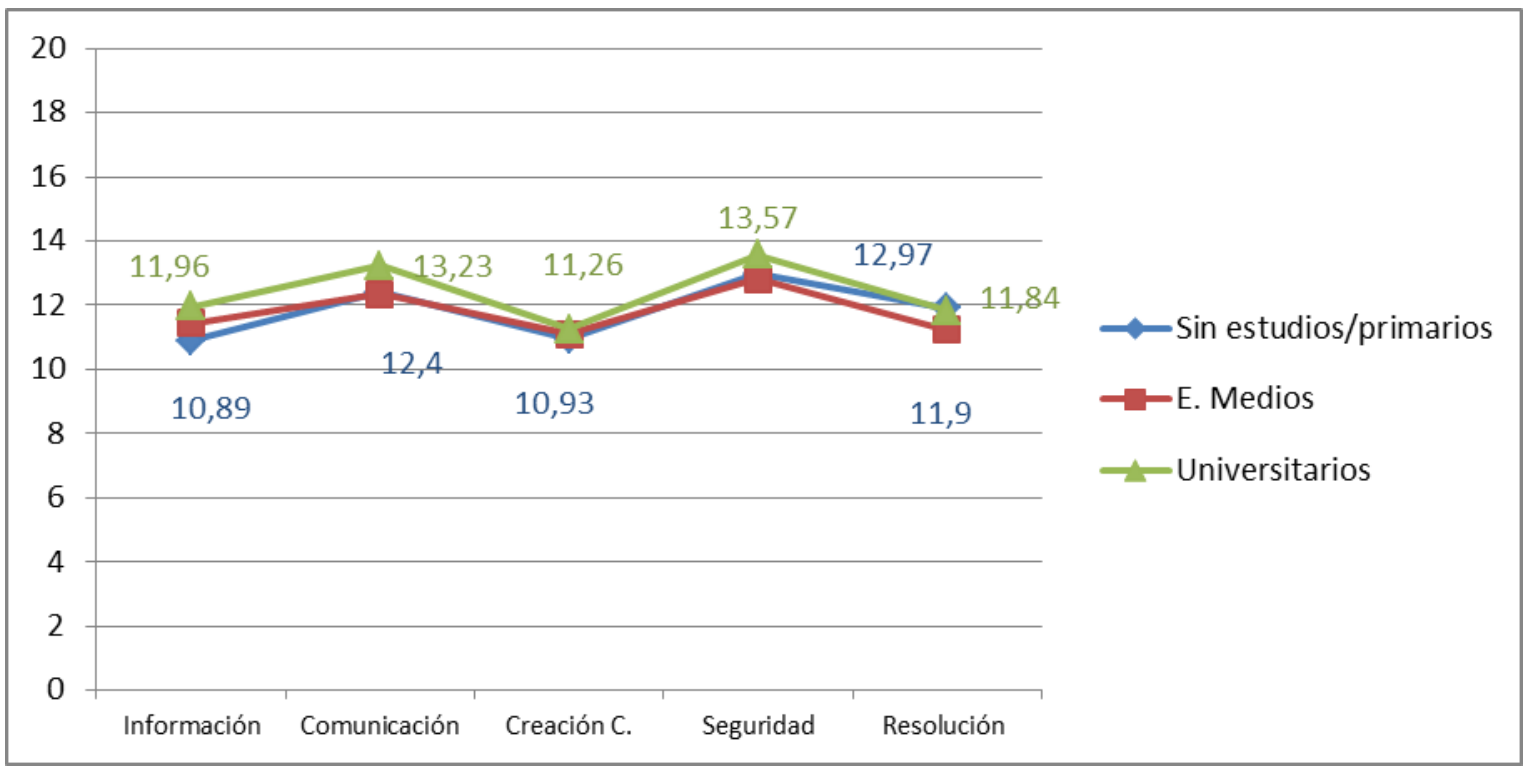

Figura 3: Media en cada área de los jóvenes según la formación de las madres. Fuente: Elaboración propia.

Así, también, cabe destacar que el alumnado que lee sin que esta actividad forme parte de las tareas escolares obtiene puntuaciones significativamente más altas en todas las áreas

Nivel de competencia digital del alumnado de educación primaria en Galicia. La influencia sociofamiliar. Esther Martínez, Adriana Gewerc y Ana Rodríguez. 
competenciales, (prueba t, nivel significación para cada área: 0,04;0,01, 0,01, 0,001, $0,05)$.

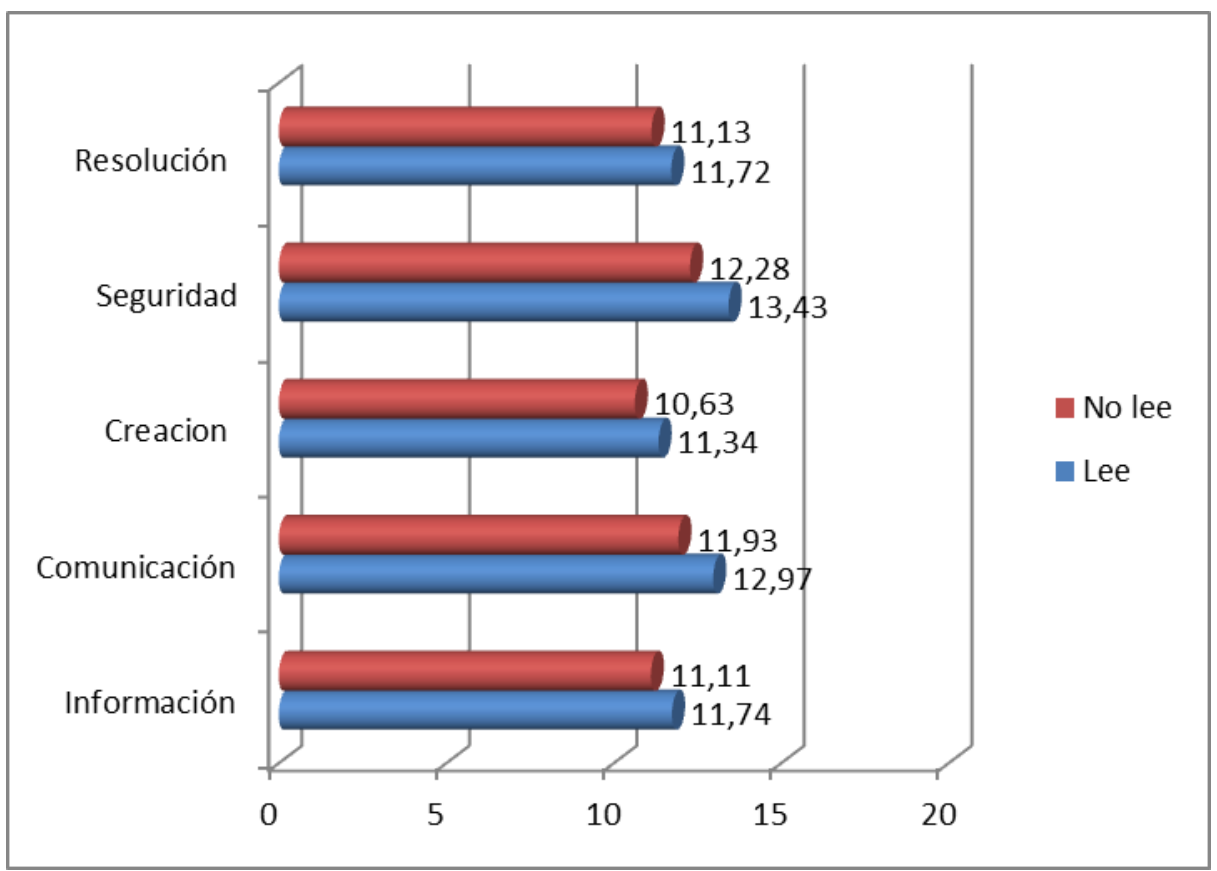

Figura 4: Media en cada área en función del hábito lector

Fuente: Elaboración propia.

En esta línea, investigaciones como las de Claro, Cabello, San Martin y Nussbaum (2015) proporcionan evidencias respecto a los profundos efectos que la situación económica, social y cultural tiene sobre la competencia digital de los estudiantes. De hecho, sus resultados muestran que el nivel educativo de los padres y madres es el factor más relevante para explicar el rendimiento del alumnado chileno en las pruebas de evaluación nacional de la competencia digital, reflejando la existencia de una segunda brecha digital en el campo de la educación.

El uso del ordenador durante los días escolares también parece tener incidencia en el desarrollo de la competencia digital, ya que la puntuación media obtenida en todas las áreas es significativamente mayor en el alumnado que emplea el ordenador con mayor frecuencia de lunes a viernes, como se representa en la gráfica. 


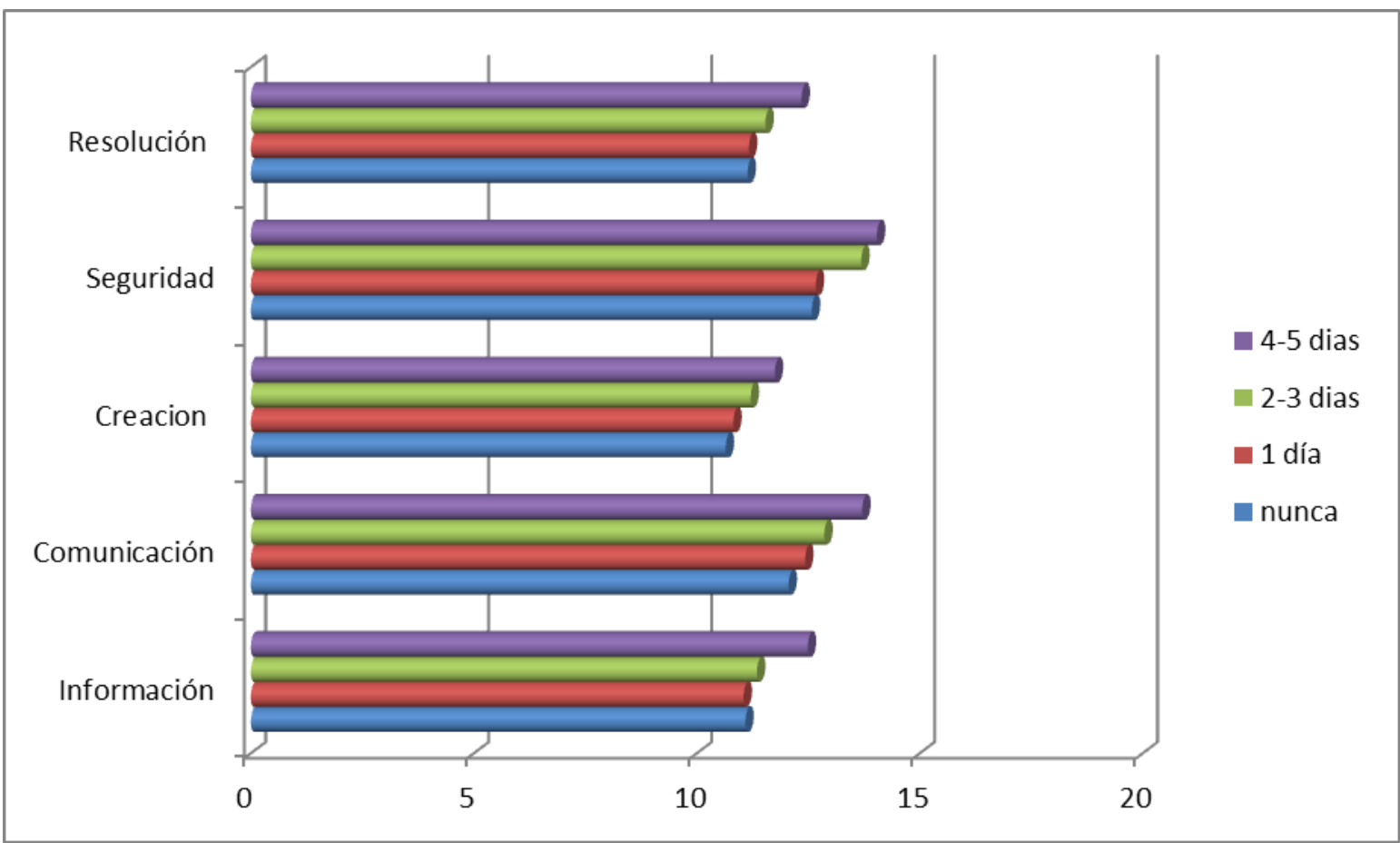

Figura 5: Puntuación obtenida en las distintas áreas en función de los días de uso del ordenador Fuente: Elaboración propia.

\section{El centro escolar}

En centros inmersos en el programa Edixgal, como cabría esperar, las medias en todas las áreas son significativamente más altas (con una significación bilateral en todos los casos de 0,000), manteniéndose seguridad y comunicación como las elevadas, pero, en este caso, es creación de contenidos y resolución de problemas las que se sitúa en último lugar, lo que parece evidenciar el escaso desarrollo curricular que estas dimensiones tienen en las aulas de estos centros educativos, a pesar de que los proyectos de disposición tecnológica en los que están involucrados buscan la adopción e integración cultural de las tecnologías en todos los ámbitos del proceso educativo (Consellería de Cultura, Educación y Ordenación Universitaria, s.f.).

Nivel de competencia digital del alumnado de educación primaria en Galicia. La influencia sociofamiliar. Esther Martínez, Adriana Gewerc y Ana Rodríguez. 


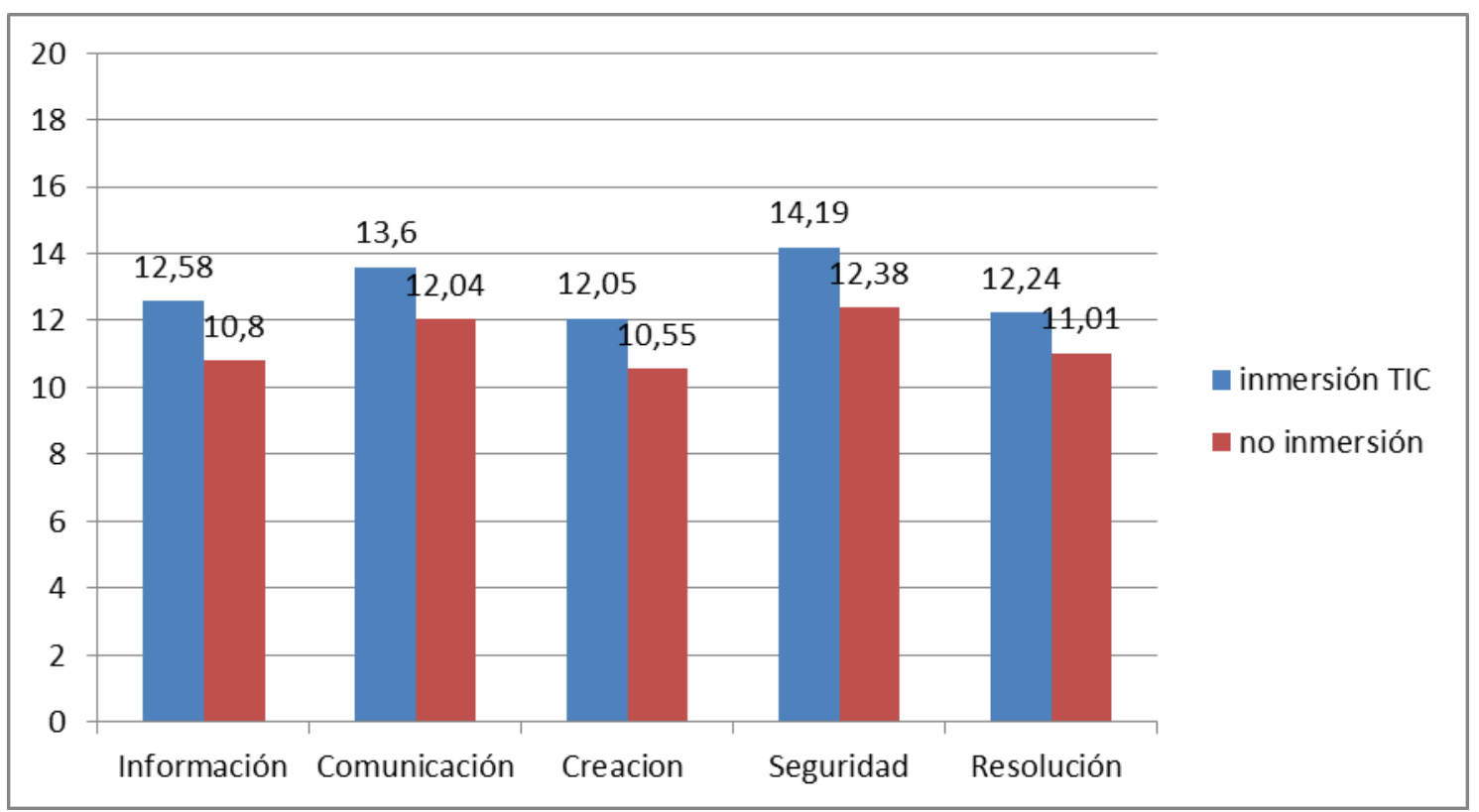

Figura 6: Media en cada área competencial del alumnado de centros con planes de inmersión TIC y centros sin planes TIC.

Fuente: Elaboración propia.

La zona también tiene cierta incidencia en el desarrollo de esta competencia. Así el alumnado de centros ubicados en ayuntamientos de tamaño medio presenta un nivel competencial más elevado en área informacional, y son las grandes ciudades las que destacan en el aspecto comunicativo $(0,047$ y 0,044 de nivel de significación respectivamente).

\section{Conclusiones}

Como hemos visto en los resultados de este trabajo, el acceso a los recursos tecnológicos no es una limitación en los preadolescentes gallegos, ya que cuentan, mayoritariamente, con ordenador y conexión a internet. Consolas, tabletas, teléfonos móviles y ordenadores son, para casi todos ellos, dispositivos habituales y utilizados de forma cotidiana. Sin embargo, el nivel competencial con estos dispositivos en términos globales no se corresponde con este hábito y se observan diferencias importantes.

Mayoritariamente, los niños y niñas obtienen un nivel medio en las cinco áreas competenciales, atendiendo de forma conjunta a conocimientos y actitudes en cada una de ellas, sin embargo, si evaluamos exclusivamente los primeros, su nivel decae, es decir, manifiestan más predisposición que capacidad real de uso. Sólo alcanzan el aprobado en las áreas de seguridad y comunicación y obtienen, en promedio, la puntuación más baja en creación de contenidos. En este contexto, el género, el nivel académico de la madre y algunos hábitos como la lectura, están mediando en el desarrollo de esta competencia. Lo que lleva a inducir que podría estar condicionado por el entorno sociocultural de pertenencia.

Nivel de competencia digital del alumnado de educación primaria en Galicia. La influencia sociofamiliar. Esther Martínez, Adriana Gewerc y Ana Rodríguez. 
Así, a través de esta investigación, podemos aventurarnos a apuntar aquellas características y condiciones que identifican a los niños y niñas con los niveles más altos de la prueba. Las niñas que tienen hábito de lectura y cuyas madres tienen estudios universitarios y aquellos/as que poseen ordenador y lo utilizan de forma cotidiana, son los que sacan puntajes más altos. Por lo que se podría hipotetizar, por un lado, que el tipo de dispositivo favorece determinadas actividades o limita otras, tal y como señalaban Salomón, Perkins y Globerson (1992) y, por otro, que el capital cultural de las familias (Bourdieu, 1986) estaría actuando como filtro para la formación en este campo ¿qué sucede en la escuela?

Los resultados obtenidos en las diferentes dimensiones reflejan dónde se encuentran las debilidades en la formación de los niños y niñas de centros públicos gallegos al finalizar la enseñanza primaria. En creación de contenidos e informacional el alumnado ha obtenido las puntuaciones más bajas. La primera tiene en cuenta aspectos como desarrollo de contenidos, su integración y reelaboración, programación y derechos de autor. La segunda (información) hace referencia a la exploración búsqueda, filtrado, almacenamiento y evaluación de la información. Ambas fundamentales para la plena participación como ciudadanos en el siglo XXI, por lo que cabría esperar que tuvieran un sitio en el desarrollo curricular de las escuelas.

El estudio de Alonso-Ferreiro (2016) expone que la dimensión informacional es la que aparece con más frecuencia en los documentos normativos curriculares (leyes, decretos que regulan la enseñanza obligatoria), pero con débil presencia en algunas áreas. En el diseño curricular de primaria predominan los contenidos referidos a la búsqueda, localización, selección y tratamiento de información en Internet y en otros soportes, considerando diferentes fuentes de información para el estudio. También hay contenidos relacionados con la recogida y el registro de datos y el desarrollo de estrategias para clasificar y recuperar, comparar, interpretar, analizar críticamente y evaluar la información. Esta abundancia de tratamiento de la dimensión en los documentos oficiales contrasta con las puntuaciones obtenidas por los niños y niñas evaluados. Cabría preguntarse en detalle, cómo se produce el desarrollo curricular en las aulas para que se genere esta problemática.

Las demás dimensiones no tienen desarrollo en las propuestas curriculares, a pesar de que los proyectos de inmersión tecnológica de la comunidad autónoma (Abalar e Edixgal) se proponen conseguir la plena alfabetización digital entre los profesores/as y alumnos/as gallegos/as. Partiendo de esta realidad, quizás los resultados de la prueba apuntan a que muchos de los aprendizajes digitales quedan en manos del contexto sociofamiliar y que este depende, a su vez, del capital cultural de las familias de origen, de sus posibilidades y limitaciones, lo que lleva a preguntarnos ¿qué sucede con los menores que no cuentan con este soporte familiar?

A través de los datos podemos atisbar que, a pesar del número de dispositivos con los que cuentan en sus hogares, e incluso la dotación en los centros educativos, existen lagunas en el conocimiento de los niños/as, fundamentalmente condicionadas por las formas de

Nivel de competencia digital del alumnado de educación primaria en Galicia. La influencia sociofamiliar. Esther Martínez, Adriana Gewerc y Ana Rodríguez. 
apropiación de los recursos tecnológicos que son diversas y que, en muchos casos, limitan el aprovechamiento de sus potencialidades (Livingston, 2017). Si las condiciones socioculturales de partida estarían orientando el desarrollo de la competencia digital, la escuela tiene un rol significativo en las compensaciones necesarias para mitigar las diferencias. Sin embargo, la escena es complicada ante una escuela que se describe como saturada de demandas y con escenarios de lo más variopintos en los hogares.

Señalaba Bolívar (2009) que "los cambios sociales en las familias han contribuido también a delegar la responsabilidad de algunas funciones educativas primarias al centro educativo" (p.7) y de manera consciente o inconsciente estas demandas son cada vez mayores, y han generado insatisfacción con su funcionamiento. Sin embargo, es necesario apelar a nuevos enfoques comunitarios "articulando la acción educativa escolar con otros ámbitos sociales, acometiendo acciones paralelas" (Bolívar, 2009, p.7) que permitan la plena inclusión de los jóvenes, independientemente de las circunstancias y contextos donde crecen.

Presentación del artículo: 14 de septiembre de 2019

Fecha de aprobación: 30 de octubre de 2019

Fecha de publicación: 30 de noviembre de 2019

Martínez-Piñeiro, E., Gewerc, A. y Rodríguez-Groba, A. (2019). Nivel de competencia digital del alumnado de educación primaria en Galicia. La influencia sociofamiliar. RED. Revista de Educación a Distancia, 61. DOI: http://dx.doi.org/10.6018/red/61/01

\section{Financiamiento}

El Proyecto de Investigación "Competencia digital en estudiantes de educación obligatoria. Entornos sociofamiliares, procesos de apropiación y propuestas de einclusión" (CDEPI) está financiado por el Ministerio de Economía y Competitividad (EDU2015-67975-C3-1-P).

\section{Referencias}

Alonso-Ferreiro, A. (2016). Competencia digital y escuela: estudio de caso etnográfico en dos CEIP de Galicia (Tesis doctoral). Universidad de Santiago de Compostela, Santiago de Compostela.

Aesaert, K., \& Van Braak, J. (2014). Exploring factors related to primary school pupils' ICT self-efficacy: A multilevel approach. Computers in Human Behavior, 41, 327-341.DOI: https://doi.org/10.1016/j.chb.2014.10.006

Aesaerta, K., Braaka, J., van Nijlenb, D., \& Vanderlindea, R. (2015). Primary school pupils' ICT competences: Extensive model and scale Development. Computers \& Education, 81, 326-344. DOI: https://doi.org/10.1016/j.compedu.2014.10.021

Nivel de competencia digital del alumnado de educación primaria en Galicia. La influencia sociofamiliar. Esther Martínez, Adriana Gewerc y Ana Rodríguez. 
Area, M., \& Sanabria, A. L. (2014). Opiniones, expectativas y valoraciones del profesorado participante en el Programa Escuela 2.0 en España. Educar, 50(1), 15-39. DOI: http://dx.doi.org/10.5565/rev/educar.64

Banaji, S. (2017). Children and media in India. London, New Delhi and New York: Routledge.

Bolívar, A. (2009). Efectos de la globalización en las vidas profesionales del profesorado. Avances en supervisión educativa, 11, 1.18. Recuperado de: https://avances.adide.org/index.php/ase/article/view/433

Bourdieu, P. (1986). The Forms of Capital. En J. Richardson (ed) Handbook of Theory and Research for the Sociology of Education, (pp. 241-256). Connecticut: Greenwood Press.

Buckingham, D. (2008). Defining Digital Literacy - What do Young People Need to Know About Digital Media?. En C. Lankshear \& M. Knobel (cords), Digital Literacies: Concepts, Policies and Practices (pp. 73-90). New York: Peter Lang.

Calvani, A., Fini, A., Ranieri, M., \& Picci, P. (2012). Are young generations in secondary school digitally competent? A study on Italian teenagers. Computers \& Education, 58(2), 797-807. DOI: https://doi.org/10.1016/j.compedu.2011.10.004

Cameron, L., Wise, S. L., \& Lottridge, S. M. (2007). The development and validation of the information literacy test. College \& Research Libraries, 68(3), 229-237.DOI: https://doi.org/10.5860/crl.68.3.229

Carbonell, X. (2014). La adicción a los videojuegos en el DSM-5. Adicciones, 26 (2). Recuperado de: https://www.redalyc.org/articulo.oa?id=289131590001

Carrera, F. X., Vaquero, E., \& Balsells, M. A. (2011). Instrumento de evaluación de competencia digital para adolescentes en riesgo social. Edutec: revista electrónica de tecnología educativa, 35, 1-25. DOI: https://doi.org/10.21556/edutec.2011.35.410

Claro, M., Cabello, T., San Martin, E., \& Nussbaum, M. (2015). Comparing marginal effects of Chilean students' economic, social and cultural status on digital versus reading and mathematics performance. Computers \& Education, 82, 1-10. https://doi.org/10.1016/j.compedu.2014.10.018

Creswell, J. W. \& Plano Clark, V. L. (2007). Designing and conducting mixed methods research. Thousand Oaks, CA, US: Sage Publications.

Consellería de Cultura, Educación y Ordenación Universitaria (s.f). Información del Proyecto Abalar: Formación y Fomento de la Cultura Digital. Xunta de Galicia. Recuperado de: https://www.edu.xunta.es/espazoAbalar/es/espazo/proxectoabalar/formacion-e-fomento-da-cultura-dixital

Corral, Y. (2009). Validez y confiabilidad de los instrumentos de investigación para la recolección de datos. Rev Ciencias de la Educación, 19 (33): 228- 247. Recuperado de: http://servicio.bc.uc.edu.ve/educacion/revista/n33/art12.pdf

Del Moral, M. E., \& Villalustre, L. (2010). Consumo televisivo y de videojuegos de los escolares asturianos versus desarrollo de competencias audiovisuales y digitales.

Nivel de competencia digital del alumnado de educación primaria en Galicia. La influencia sociofamiliar. Esther Martínez, Adriana Gewerc y Ana Rodríguez. 
En E. del Moral. (Coor.), Televisión: desarrollo de la creatividad e infancia (pp. 31-46). Barcelona, España: Octaedro.

Dornaleteche, J.; Buitrago, A. \& Moreno, L. (2015). Categorización, selección de ítems y aplicación del test de alfabetización digital online como indicador de la competencia mediática. Comunicar, 22(44), 177-185. DOI: https://doi.org/10.3916/C44-2015-19

Fain, M. (2011) Assessing Information Literacy Skills Development in First Year Students: A Multi-Year Study. The Journal of Academic Librarianship, 37(2), 109-119. DOI: http://dx.doi.org/10.1016/j.acalib.2011.02.002

Ferrari, A. (2012). Digital Competence in Practice: An Analysis of Frameworks. Luxembourg: Publications Office of the European Union.

Ferrés, J., García, A., Aguaded, J. I., Fernández, J., Figueres, M., Blanes, M., ... Zarandona, E. (2011). Competencia mediática. Investigación sobre el grado de competencia de la ciudadanía en España. Madrid: INTEF-Ministerio de Educación. Disponible en: http://ntic.educacion.es/w3/competencia_mediatica/competencia_mediatica.pdf

Fraga-Varela, F., \& Alonso-Ferreiro, A. (2016). Presencia del libro de texto digital en Galicia: Una mirada estadístico-geográfica del proyecto E-DIXGAL.

Profesorado: Revista de Currículum y Formación de Profesorado, 20 (1), 91112. Recuperado de: https://recyt.fecyt.es//index.php/profesorado/article/view/49870

García, R.; Duarte, A., \& Guerra, S. (2014). Propuesta de un instrumento de evaluación para medir el grado de competencia mediática en la etapa de la educación infantil. Pixel-Bit: Revista de medios y educación, 44, 81-96.

DOI: http://dx.doi.org/10.12795/pixelbit.2014.i44.06

George, D. \& Mallery, P. (2006). Spss for Windows step by step: A Simple Guide and Reference. 13.0 ( $6^{\mathrm{a}}$ ed.). Boston: Pearson Education, Inc.

Gewerc, A., \& Montero, M. L. (2013). Culturas, formación y desarrollo profesional. La integración de las TIC en las instituciones educativas. Revista de Educación, 362, 323-347. DOI: http://dx.doi.org/10-4438/1988-592X-RE-2011-362-163

Gisbert, M.; Espuny, C., \& González, J. (2011). INCOTIC. Una herramienta para la @utoevaluación diagnóstica de la competencia digital en la universidad.

Profesorado: Revista de curriculum y formación del profesorado, 15(1), 75-

90. Recuperado de:

https://recyt.fecyt.es/index.php/profesorado/article/view/42011

González, J., Espuny, C., de Cid. M. J., \& Gisbert, M. (2012). INCOTICESO. Cómo autoevaluar y diagnosticar la competencia digital en la Escuela 2.0. Revista de Investigación Educativa, 30(2), 287-302. DOI: https://doi.org/10.6018/rie.30.2.117941

Imison, C., Castle-Clarke, S., Watson, R., \& Edwards, N. (2016). Delivering the benefits of digital health care. Nuffield Trust. Recuperado de: https://www.nuffieldtrust.org.uk/research/delivering-the-benefits-of-digitalhealth-care

Nivel de competencia digital del alumnado de educación primaria en Galicia. La influencia sociofamiliar. Esther Martínez, Adriana Gewerc y Ana Rodríguez. 
INE (2018). Encuesta sobre equipamiento y uso de tecnologías de información y comunicación en los hogar. Instituto Nacional de Estadística. Recuperado de: https://www.ine.es/prensa/tich_2018.pdf

Iordache, C., Mariën, I., \& Baelden, D. (2017). Developing Digital Skills and Competences: A Quick- Scan Analysis of 13 Digital Literacy Models. Italian Journal of Sociology of Education, 9(1), 6-30. DOI: https://doi.org/10.14658/pupj-ijse-2017-1-2

Jones, R. H., \& Hafner, C. A. (2012): Understanding digital literacies. A practical introduction. NY: Routledge.

Katz, I. R. (2007). Testing information literacy in digital environments: ETS's iSkills assessment. Information technology and Libraries, 26(3), 3-12. DOI: https://doi.org/10.6017/ital.v26i3.3271

Kleine, D., Hollow, D., \& Poveda, S. (2014). Children, ICT and development: Capturing the potential, meeting the challenges. Florence: UNICEF Office of Research-Innocenti. Recuperado de: www.unicefirc.org/publications/pdf/unicef_royalholloway_ict4dreport_final.pdf

Lloyds Bank (2016). Consumer Digital Index - Benchmarking the Digital and Financial Capability of UK Consumers. Recuperado de: http://www.lloydsbank.com/assets/media/pdfs/ConsumerDigitalIndex_280715A P_0116_v9_Web.pdf.

Lacasa, P. (2011). Los videojuegos. Aprender en mundos reales y virtuales. Madrid: Morata.

Lawshe, C. H. (1975). A quantitative approach to content validity. Personnel Psychology, 28(4), 563-575.

Livingstone, S., Haddon, L., \& Görzig, A. (eds). (2012). Children, risk and safety on the internet: Research and policy challenges in comparative perspective. Bristol: Policy Press. Recuperado de: http://eprints.lse.ac.uk/44761/

Livingstone, S., Nandi, A., Banaji, S., \& M. Stoilova. (2017). Young Adolescents and Digital Media Uses, Risks and Opportunities in Low-and Middle-Income Countries: A Rapid Evidence Review. London: GAGE

LOE. Ley Orgánica 2/2006, de 3 de mayo, de Educación (2006).

LOMCE. Ley Orgánica 8/2013, de 9 de diciembre, para la mejorar de la calidad educativa (2013).

López-Mondéjar. L. (2018). El uso de la televisión en el hogar y en el aula de Educación Primaria. Educatio Siglo XXI, 36 (1), 195-214.

DOI: https://doi.org/10.6018/j/324231

NIH Office of Behavioral and Social Sciences. (2018). Best practices for mixed methods research in the health sciences ( 2 nd ed). Bethesda: National Institutes of Health.

Marco, C., \& Chóliz, M. (2014). Tratamiento cognitivo-conductual de la adicción a videojuegos de rol online: fundamentos de propuesta de tratamiento y estudio de

Nivel de competencia digital del alumnado de educación primaria en Galicia. La influencia sociofamiliar. Esther Martínez, Adriana Gewerc y Ana Rodríguez. 
caso. Anales de psicología, 30(1). DOI:

http://dx.doi.org/10.6018/analesps.30.1.150851

McDougall, J., Readman, M., \& Wilkinson, P. (2018). The uses of (digital) literacy. Learning, Media and Technology, 43 (3), 263-279. DOI: https://doi.org/10.1080/17439884.2018.1462206

Montero, L. M., \& Gewerc, A. (Coords.) (2013). Una historia, cuatro historias. Acompañar proyectos de innovación educativa con las TIC. Barcelona: Graó.

Oakleaf, M. (2008). Dangers and opportunities. A conceptual map of information literacy assessment approaches. Libraries \& the Academy, 8, 233-253. Recuperado de: http://meganoakleaf.info/dangersopportunities.pdf

OCDE (2014). Education at a Glance 2014. OECD indicators. OECD Publishing. DOI: http://dx.doi.org/10.1787/eag-2014-en

Pérez-Escoda, A., Castro-Zubizarreta, A., \& Fandos-Igados, M. (2016). La competencia digital de la Generación Z:claves para su introducción curricular en la Educación Primaria. Comunicar, 14(49). DOI: http://dx.doi.org/10.3916/C49-2016-07

Pluye, P. \& Hong, Q.N. (2014). Combining the Power of Stories and the Power of

Numbers: Mixed Methods Research and Mixed Studies Reviews. Annual Review of Public Health, 35, 29-45. DOI: https://doi.org/10.1146/annurev-publhealth032013-182440

Pinto, M. (2009). Design of the IL-HUMASS survey on information literacy in higher education: A self-assessment approach. Journal of Information Science, 36 (1), 86-103. DOI: http://dx.doi.org/10.1177/0165551509351198

Salomon. G., Perkins, D., \& Bloberson, T., y(1992). Coparticipando en el conocimiento: la ampliación de la inteligencia humana con las tecnologías inteligentes. $C L \&$ E: Comunicación, lenguaje y educación, 13, 6-22. Recuperado de: https://dialnet.unirioja.es/servlet/articulo?codigo $=126248$

Sancho, J.M., \& Alonso-Cano, C. (2012) La fugacidad de las políticas, la inercia de las prácticas: La educación y las tecnologías de la información y la comunicación. Barcelona: Ocatedro.

Sedeño, A. (2010). Videojuegos como dispositivos culturales: las competencias espaciales en educación. Comunicar, 17 (34), 183-189, http://dx.doi.org/10.3916/C34-2010-03-018

Tristán-López, A. (2008). Modificación al modelo de Lawshe para el dictamen cuantitativo de la validez de contenido de un instrumento objetivo. Avances en medición, 6, 37-48. Recuperado de: https://dialnet.unirioja.es/servlet/articulo?codigo=2981185

UNECOSOC (United Nations Economic and Social Council) (2015). Digital development: Report of the Secretary- General. Eighteenth Session of the Commission on Science.

UNICEF (2013). Integrating ICTs into communication for development strategies to support and empower marginalized adolescent girls. New York: UNICEF. Recuperado de: www.unicef.org/cbsc/files/ICTPaper_Web.pdf

Nivel de competencia digital del alumnado de educación primaria en Galicia. La influencia sociofamiliar. Esther Martínez, Adriana Gewerc y Ana Rodríguez. 
UNICEF (2017). Estado Mundial de la Infancia: Niños en un mundo digital. New York: UNICEF. ISBN 978-92-806-4940-6. Recuperado de: https://www.unicef.org/media/48611/file

Van Deursen, A., \& Van Dijk, J. (2011). Internet skills and the digital divide. New media \& Society, 13(6), 893-911, DOI: http://dx.doi.org/10.1177/1461444810386774.

Van Deursen, A; \& Van Diepen, S. (2013). Information and strategic Internet skills of secondary students: A performance test. Computers \& Education 63, 218-226. DOI: https://doi.org/10.1016/j.compedu.2012.12.007

Van Deursen, A. J., Görzig, A., Van Delzen, M., Perik, H. T. \& Stegeman, A. G. (2014). Primary School Children's Internet Skills: A Report on Performance Tests of Operational, Formal, Information, and Strategic Internet Skills. International Journal of Communication, 8, 1327-1349. Recuperado de: https://ijoc.org/index.php/ijoc/article/view/2407

Van Dijk, J., \& Van Deursen, A. (2014). Digital skills: unlocking the information society (First edition). New York, NY: Palgrave Macmillan.

Villa, A., \& Poblete, M. (2011). Evaluación de competencias genéricas: Principios, oportunidades y limitaciones. Bordón, 63(1), 147-170. Recuperado de: https://www.upv.es/entidades/ICE/info/EvaluacionCompetenciasGenericas.pdf

Vuorikari, R., Punie, Y., Carretero, S., \& Van den Brande, L. (2016). DigComp 2.0: The Digital Competence Framework for Citizens. Update Phase 1: The Conceptual Reference Model. Luxembourg: Publication Office of the European Union. DOI: https://doi.org/10.2791/11517

WEF (World Economic Forum) (2015). Global information technology report. Recuperado de: http://reports.weforum.org/global-information-technologyreport-2015/

World Bank (2016). World Development Report: Digital Dividends. Washington, DC: World Bank. Recuperado de: http://documents.worldbank.org/curated/en/896971468194972881/pdf/102725PUB-Replacement- PUBLIC.pdf.

Zhong, Z. (2011). From access to usage: The divide of self-reported digital skills among adolescents. Computers \& Education, 56, 736-746. DOI: https://doi.org/10.1016/j.compedu.2010.10.016

Nivel de competencia digital del alumnado de educación primaria en Galicia. La influencia sociofamiliar. Esther Martínez, Adriana Gewerc y Ana Rodríguez. 